'Unidad de Tratamiento del Ataque Cerebrovascular (UTAC), Servicio de Neurología, Departamento de Medicina, Clínica Alemana de Santiago. Facultad de Medicina Clínica

Alemana-Universidad del Desarrollo. Santiago, Chile.

${ }^{2}$ Servicio de Neurología, Hospital de Valdivia. Valdivia, Chile Departamento de Neurología, Universidad Austral de Valdivia. ${ }^{3}$ Servicio de Neurología, Unidad de Tratamiento del Ataque Cerebrovascular (UTAC), Clínica Alemana de Temuco, Temuco, Chile. Departamento de Especialidades, Unidad de Neurología, Universidad de La Frontera, Temuco, Chile.

Fuentes de apoyo de financiamiento: Ninguno

Recibido el 8 de marzo de 2014, aceptado el 10 de septiembre de 2014

Correspondencia a: Dr. Alejandro Brunser R. Servicio de Neurología, Clínica Alemana de Santiago. Manquehue Norte $1410,10^{\circ}$ piso, Vitacura Santiago, Chile. Fax: 56-2-2101061 abrunser2002@yahoo.com

\section{Sonotrombolisis en el ataque cerebrovascular isquémico: once años de experiencia en Clínica Alemana de Santiago}

\author{
ALEJANDRO BRUNSER ${ }^{1}$, ARNOLD HOPPE ${ }^{1}$, PAULA MUÑOZ $^{1}$, \\ DANIEL CÁRCAMO ${ }^{1}$, PABLO M. LAVADOS ${ }^{1}$, JAVIER GAETE $^{1}$, \\ ANDRÉS ROLDÁN ${ }^{2}$, RODRIGO RIVAS ${ }^{3}$
}

\section{Sonothrombolysis for acute ischemic stroke}

Background: Sonothrombolysis (ST) is an emerging modality for the treatment of stroke. Aim: To assess the feasibility to perform ST in a Chilean hospital. Material and Methods: Patients attended at a private clinic with an acute ischemic stroke, between September 2002 and May 2013 and eligible for endovenous thrombolysis, were studied with a transcranial Doppler (Spencer PMD 100 or $\left.150^{\circledR}\right)$. Those with an adequate sonographic window and a demonstrated arterial occlusion were monitored continuously with transcranial Doppler at the site of worst residual flow following the CLOTBUST study protocol. Results: One thousand twenty six patients were studied, of whom 136 received intravenous thrombolysis ( $r t-P A)$ and 61, aged $66 \pm 18$ years (59\% males), were subjected to ST (7\% of total). Their median National Institutes of Health Stroke Scale score was 14, the lapse from symptom onset to rt-PA was 127 minutes (43-223). Middle cerebral artery (MCA) occlusion was found in $88.5 \%$ of patients. Complete recanalization was achieved in $44.3 \%$ of patients. Sixty percent had Modified Rankin Scale of 0 to 2 at 3 months (95\% confidence intervals (CI) 48.1 to 72). Case fatality was $9.8 \%$ and asymptomatic intracranial hemorrhage occurred in 9.8\% (95\% CI: 4.3 to 20.2). Conclusions: ST can be carried out in a complex medical center and is safe.

(Rev Med Chile 2014; 142: 1238-1244)

Key words: Stroke; Therapy; Thrombolytic Therapy; Ultrasonography, Doppler.
4 n Chile, la enfermedad cerebrovascular es una de las principales causas de muerte, con una tasa de mortalidad de 52 por 100.000 habitantes el 2010. Es la principal causa de muerte en las mujeres, ya desde el $2000^{1}$.

$\mathrm{El}$ ataque cerebrovascular agudo isquémico (ACVi) representa aproximadamente 65\% de los ACV agudos en Chile y es causa de discapacidad, dolor crónico, depresión y demencia ${ }^{2}$.

La trombolisis endovenosa (TEV) hasta las 4,5 horas de evolución es la única terapia aguda aprobada para esta patología ${ }^{3,4}$. Si bien este tratamiento es capaz de aumentar en $30 \%$ la oportunidad de tener mínimas o ninguna secuela comparado con el placebo, su capacidad para recanalizar grandes arterias cerebrales ocluidas es baja, no superando 15 a $20 \%{ }^{5}$. Alternativas terapéuticas para mejorar estos rendimientos, como la recanalización intraarterial, no han demostrado ser superiores en cuanto al desenlace ${ }^{6-8}$ y el uso de nuevos trombolíticos más potentes aún se encuentra en estudio ${ }^{9,10}$.

La TEV asociada al uso de ondas de ultrasonido (US) del tipo del Doppler transcraneal (DTC) o dúplex transcraneal ha demostrado resultados 
promisorios en cuanto al incremento de las tasas de reperfusión arterial y eventualmente al pronóstico de los pacientes ${ }^{11,12}$. La energía transmitida por las ondas de ultrasonido produce fenómenos de fisuración y cavitación del trombo, lo que permitiría mejorar la penetración del agente fibrinolítico al interior del trombo, resultando en una mayor recanalización arterial ${ }^{13}$. El uso del DTC durante la TEV permite, además, documentar el éxito o fracaso del tratamiento endovenoso en cuanto a la recanalización arterial y a la derivación selectiva de pacientes a una terapia de rescate con técnicas de tipo intraarteriales ${ }^{14}$ dentro de los estrechos márgenes de tiempo que la terapia de la isquemia cerebral focal impone.

Los reportes de experiencias de sonotrombolisis con rt-PA (ST) (combinación de trombolítico y ultrasonido) son escasos a nivel mundial y provienen de países con alto nivel de desarrollo ${ }^{11,12,15}$. La factibilidad de realizar ST en nuestro medio y sus resultados no ha sido extensamente evaluada.

\section{Pacientes y Métodos}

En el servicio de urgencia de Clínica Alemana de Santiago (SUCAS), el procedimiento protocolizado ante la sospecha de un paciente con un infarto cerebral en tiempo de TEV, es la activación de un código ACV, llamado "azul”, en éste, un equipo de tomografía axial computada (TAC) es dejado libre y el neurólogo de turno acude a la brevedad, evalúa el déficit neurológico del paciente por medio de la escala de ACV del National Institute of Health (NIHSS) ${ }^{16-19}$, se obtienen los datos demográficos, se toman exámenes generales de sangre: recuento globular, TP/INR, TTPK, glicemia, creatinina y ELP. El enfermo es sometido posteriormente a un protocolo de imágenes estandarizado que consiste en: una tomografía axial computada de cerebro sin contraste, una angio-TAC de vasos cervicales y cerebrales y una difusión cerebral por resonancia nuclear magnética $(\mathrm{RNM})^{20}$. En enfermos alérgicos al medio de contraste la angio-TAC puede ser reemplazada por una angiografía por RNM. Si el paciente es candidato a TEV, el bolo del trombolítico es administrado generalmente luego de la TAC. Posterior a estas imágenes se realiza un DTC diagnóstico por un neurosonografista de turno. Entre los años 2002 y 2006, los turnos fueron cubiertos por 2 neurólogos y posteriormente por 4 , quienes hacen turnos semanales. Para la realización de dicho examen se utiliza un DTC, Spencer Technologies ${ }^{\circledR}$ (PMD 100 ó 150) con un transductor de 2-MHz y parámetros de adquisición: repetición de pulso frecuencia 8 $\mathrm{KHz}$, ciclo, 6,4\%, intensidad promedio de máximo peak espacial $238 \mathrm{~mW} / \mathrm{cm}^{2}$; e índice mecánico 0,24 , utilizando para esto un protocolo institucional ${ }^{21}$.

En caso de obstrucción arterial al DTC, ésta es graduada por la escala de TIBI, (Thrombolysis in Brain Ischemia flow $)^{22}$, que puntúa el estado vascular en isquemias cerebrales en 6 puntos (Figura 1). Las curvas de tipo TIBI 0 a 3 son consideras obstructivas, mientras las de tipo las curvas TIBI 4 y 5 , corresponden a un vaso abierto. Aquellos pacientes que tienen una ventana sonográfica suficiente y demuestran una oclusión arterial al ultrasonido son monitorizados con DTC continuo en el sitio de peor flujo residual siguiendo el protocolo del estudio CLOTBUST ${ }^{11}$.

De existir buena ventana acústica, el monitoreo de las arterias de la circulación anterior se realiza con un casco Marc 500 (Spencer Technologies) que mantiene fija la zona de insolación, de ser la ventana difícil o comprometer vasos de la circulación vertebro-basilar, este monitoreo se realiza a mano.

Se consideró recanalización completa el paso de cualquier señal obstructiva de TIBI ( 0 a 3 ) a una señal abierta TIBI (4 ó 5) con mantención de ésta a las $2 \mathrm{~h}$ post bolo de trombolítico al DTC. Se definió como reapertura parcial al aumento de 1 punto o más en la escala de TIBI de una señal oclusiva sin llegar a puntajes de 4 ó 5 a las 2 h. Finalmente, la ausencia de recanalización se definió como la ausencia de cambio del TIBI inicial al final del monitoreo. Todos los pacientes post trombolisis fueron hospitalizados en la Unidad de Tratamientos Intensivos o Unidad de Tratamiento del Ataque Cerebral (UTAC) de Clínica Alemana de Santiago y fueron estudiados con TAC cerebral de control a las 22-36 h o en caso de deterioro clínico.

Se consideró hemorragia cerebral sintomática a una lesión hiperdensa nueva asociada a un deterioro neurológico del paciente en 4 puntos o más en la escala de NIHSS, en las primeras $72 \mathrm{~h}$ de evolución y sin otra causa que lo explique.

El estado funcional del paciente se midió a los tres meses de su alta según modified Rankin Scale $(\mathrm{mRS})^{23-25}$, en una entrevista médica presencial. Se definió como independiente aquellos pacientes que presentaban a 3 meses un mRS de 0 a 2 y como dependiente los pacientes con puntajes de 3 a 5 . 
En este estudio descriptivo de casos clínicos de ACVi tratados con ST, los datos fueron obtenidos en forma retrospectiva del registro prospectivo de enfermedades cerebrovasculares de Clínica Alemana (RECCA), aprobada por el comité de ética Cínica Alemana Santiago-Universidad del Desarrollo y que consta de consentimiento informado escrito.

El objetivo principal del estudio es describir la factibilidad de realizar ST en nuestro centro.

Los objetivos secundarios fueron indicadores de eficacia como la recanalización del vaso ocluido, la seguridad que fue evaluada como la hemorragia intracraneal, según criterios de ECASS III (4) y el desenlace a los 3 meses por medio de la modified Rankin Scale.

\section{Análisis estadístico}

Se calculó el porcentaje de pacientes trombolizados entre septiembre de 2002 y mayo de 2013 y de estos cuantos fueron susceptibles a ST. En este grupo describimos porcentajes de reapertura arterial, completa, parcial y nula. El porcentaje de complicaciones hemorrágicas intracraneanas y describimos el mRS a 3 meses. Nuestros valores fueron comparados con los del estudio más grande de ST en la actualidad, CLOTBUST. Se realizó un análisis de "cohorte de supervivencia", por lo que se calculó la reducción relativa del riesgo (RRR) y reducción absoluta de riesgo (RAR) para un mal desenlace $(m R S \geq 3)$ en aquellos enfermos que presentaron reperfusión total o parcial a $2 \mathrm{~h}$. Los intervalos de confianza (IC 95\%) para proporciones se obtuvieron por el método Wald ${ }^{26}$.

\section{Resultados}

Durante el período de estudio, ingresaron consecutivamente a SUCAS, 1.126 pacientes con enfermedad cerebro vascular isquémica, de los cuales $136(12 \%)$ fueron a TEV, del total, 79 pacientes $(59,8 \%)$ demostraron oclusiones vasculares en el estudio inicial de polígono arterial. De éstos, 61 pacientes (7\% del total) recibieron ST; las causas de exclusión de 18 pacientes fueron: en 3 casos el neurosonólogo de turno no fue llamado (el pronóstico a 3 meses de estos pacientes por mRS fue de 0,1 y 4 ), en 2 casos se encontró al momento del DTC un TIBI 4 en la arteria afectada (ambos en mRS en 0 a los 3 meses), en 1 caso la calidad del monitoreo fue insuficiente, pues por

\section{Tabla 1. Características de los enfermos} sonotrombolizados

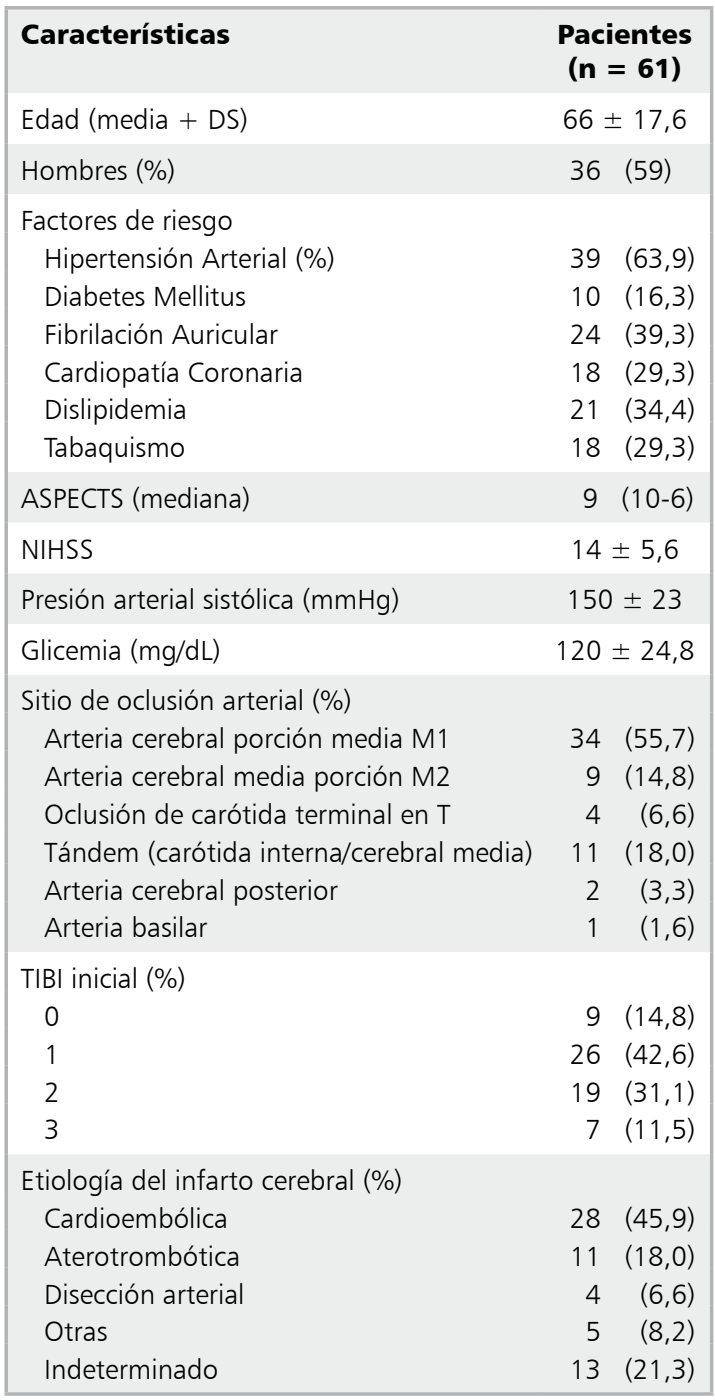

la gravedad del paciente y extensión del trombo se planeó desde el inicio una trombectomía (en mRS 1 a los 3 meses), 11 enfermos no tenían ventana acústica, todos estos enfermos fueron mujeres, con un promedio de edad de 84,4 años (79-96) y pronóstico menos favorable, solamente 3 pacientes obtuvieron un mRS de 0 a 2 .

Las características de los enfermos con ST (Tabla 1) fueron: 37 pacientes presentaban al ingreso un NIHSS superior a 10 puntos $(60,1 \%)$ y $14(23 \%)$ superior a 20 puntos. La mediana de 


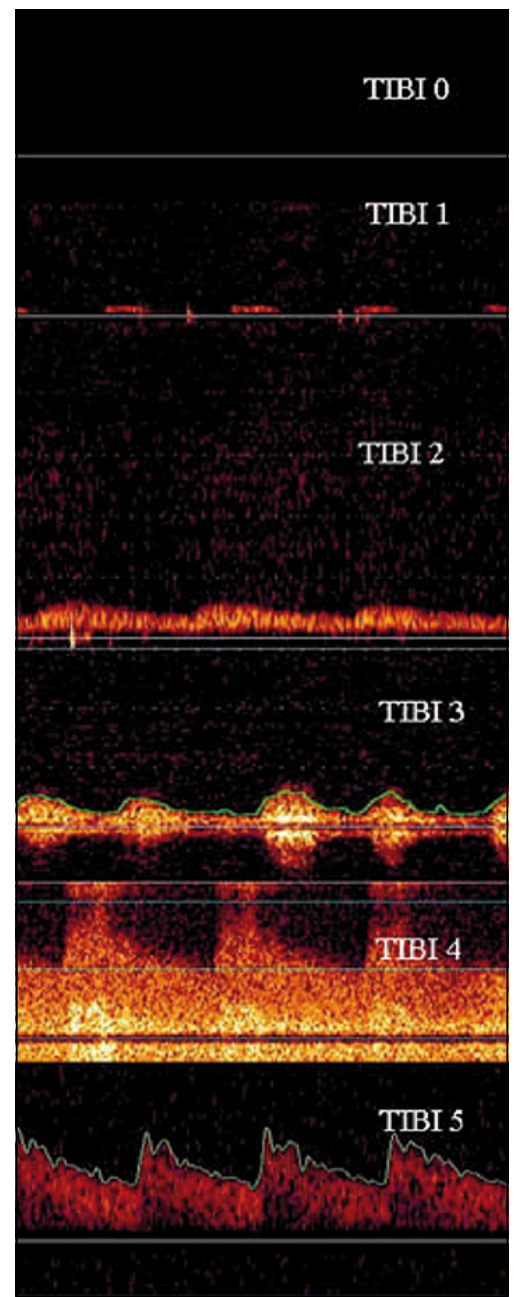

Figura 1. TIBI 0 : ausencia absoluta de curvas de velocidad de flujo en el DTC. TIBI 1: presencia sólo de flujo en la sístole. TIBI 2: curva de baja velocidad y aceleración lenta de la sístole con baja resistencia. TIBI 3: curva de baja velocidad y resistencia alta. TIBI 4: curva de alta velocidad o estenótica. TIBI 5: curva normal.

tiempo desde inicio del ACVi a bolo de rt-PA fue de $127 \mathrm{~min}$ (43-223), con 13 (21,3\%) tratados antes de $90 \mathrm{~min}$. La dosis promedio de trombolítico fue de 60,5 mg (90-12). Luego de $2 \mathrm{~h}$ con el DTC se demostró repermeabilización total arterial en $27(44,3 \%)$ casos, repermeabilización parcial en $10(16,4 \%)$ enfermos y ausencia de cualquier recanalización en $24(39,3 \%)$ de los tratados. A 14 pacientes se les ofreció la posibilidad de rescate con terapias intraarteriales, de éstos, $9(64,2 \%)$ estaban independientes a los 3 meses. En la Figura 2, se

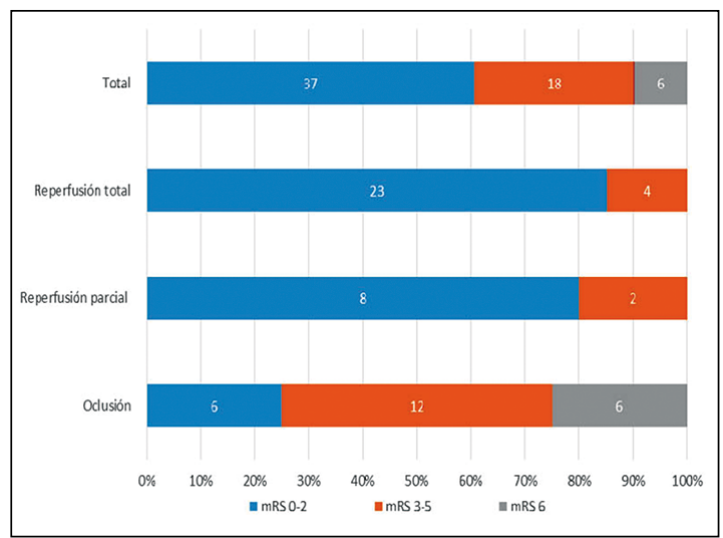

Figura 2. Desenlace según grado de reperfusión por sonotrombolisis.

observa que los pacientes con recanalización arterial, ya sea parcial o completa obtienen una mayor proporción de pacientes independientes, menor proporción de dependientes y de mortalidad, este último desenlace sólo ocurrió en pacientes en los que se mantuvo el vaso ocluido. Se redujo en $78 \%$ la posibilidad de un mal desenlace (mRS $\geq 3$ ), en caso de reperfusión vascular total o parcial (reducción relativa del riesgo; RRR: 0,78, IC 95\%; 0,53-0,90). Los pacientes expuestos al vaso ocluido tuvieron un riesgo relativo (RR) de 4,63 (IC 95\%; 2,15-9,97), de tener un mal desenlace. La reducción absoluta de riesgo (RAR) fue de 0,59 (IC 95\%; 0,38-0,80).

En 6 casos de pacientes tratados se presentó una hemorragia cerebral sintomática, de éstos, 3 estaban independientes a lo 3 meses y los otros severamete discapacitados o muertos.

De los 61 pacientes tratados, $37(60,6 \%)$ estaban independientes a los 3 meses, 18 (29,5\%) estaban discapacitados y $6(9,8 \%)$ habían fallecido.

La Tabla 2 compara nuestros resultados con los del estudio de CLOTBUST, incluyendo los grupos de pacientes trombolizados sólo endovenosamente y adicionalmente con ST.

\section{Discusión}

La TEV, es una terapia efectiva que mejora el pronóstico de los enfermos portadores ACVi tratados a 3 meses, aún en aquellos que poseen grandes arterias cerebrales ocluidas. Un metaanálisis y una reciente revisión sistemática con me- 
Tabla 2. Seguridad y eficacia comparación al estudio CLOTBUST

\begin{tabular}{|lccc|}
\hline & $\begin{array}{c}\text { CLOTBUST control } \\
\text { (rt-PA) }\end{array}$ & $\begin{array}{c}\text { CLOTBUST tratamiento } \\
\text { (ST+rt-PA) }\end{array}$ & $\begin{array}{c}\text { Clínica Alemana Santiago } \\
\text { (ST+rt-PA) }\end{array}$ \\
Número de pacientes & 63 & 63 & 61 \\
Edad & $70 \pm 13$ & $67 \pm 12$ & $66 \pm 18$ \\
NIHSS pre bolo & 17 & 16 & 14 \\
Tiempo a bolo & 130 & 150 & 127 \\
Oclusión arteria cerebral media & $100 \%$ & $100 \%$ & $88,5 \%$ \\
Recanalización completa & $17,6 \%$ & $46 \%$ & $44,3 \%$ \\
mRS 0-2 a tres meses & $36,7 \%$ & $50,9 \%$ & $60,6 \%$ \\
Hemorragia cerebral sintomática & (IC: $95 \% ; 25-51 \%)$ & $($ IC: $95 \% ; 38-64 \%)$ & $($ IC: $95 \% ; 48-72 \%)$ \\
\hline
\end{tabular}

todología Cochrane concluyó que la ST realizada en pacientes con ACVi agudo de arteria cerebral media y cerebral posterior produce un aumento de la recanalización, sin aumento significativo de la transformación hemorrágica del infarto cerebral. Además, existió una mejoría clínica estadísticamente significativa a los tres meses, en términos de muerte y discapacidad, al incorporar US de baja frecuencia a enfermos tratados con trombolíticos, mejorand, el porcentaje de recanalización arterial y no aumentando la hemorragia cerebral sintomática, comparado con trombolítico endovenoso sólo ${ }^{27,28}$.

En nuestra experiencia, y por un plazo de 4 años los monitoreos de ST, fueron realizados solamente por 2 operadores, posteriormente la cantidad de operadores fue aumentada a 4 . En el plazo de esta experiencia, solamente 3 enfermos no fueron trombolizados con monitoreo y esto se debió a no ser informado el neurosonólogo de turno. Una experiencia realizada en Clínica Alemana Temuco demuestra que un centro neurovascular de menor complejidad y con un sólo operador de ultrasonografía, fue capaz de realizar todos los monitoreos ultrasonográficos, obteniendo similares resultados ${ }^{29}$.

Respecto a nuestros resultados clínicos, estos son muy similares a los del estudio CLOTBUST, un estudio aleatorizado ciego, el más grande realizado en ST (Tabla 2). El porcentaje de recanalización arterial completa es prácticamente el mismo, superando $40 \%$ de los tratados en el estudio CLOTBUST. La reperfusión arterial temprana se relacionó con un clara menor posibilidad de quedar discapacitado o morir, tal como lo muestra el metanálisis de $\mathrm{Rha}^{27}$ en pacientes con infarto cerebral; la reperfusión temprana incrementa en 5 veces la posibilidad de que un paciente sea autovalente y reduce en 4 veces la posibilidad de muerte.

En cuanto al pronóstico a 3 meses definido como un mRS 0-2 a tres meses, nuestros resultados también fueron similares al del estudio CLOTBUST, con sobre $50 \%$ de los pacientes independientes.

La ST permitió, además, el rescate de pacientes que no presentaron apertura arterial post trombolitico endovenoso mediante técnicas de revascularización intraarterial, con un buen pronóstico a 3 meses en sobre $60 \%$ de ellos.

Por último, en nuestro trabajo observamos hemorragia sintomática en $9,8 \%$ de los casos, mayor al descrito en el estudio CLOTBUST, de $4,8 \%$, sin embargo, este se encuentra dentro del rango de dispersión de los intervalos de confianza. En nuestro estudio casi un cuarto de los pacientes tenían extensos infartos clínicos, además de ser realizadas 12 trombolisis $(19,6 \%)$ después de las 3 $\mathrm{h}$ de inicio clínico, los cuales tienen mayor riesgo de transformación hemorrágica.

Nuestro estudio posee fortalezas como haber sido realizado en una instancia clínica habitual y con un número importante de enfermos, logrando completar todos los pacientes con los tres meses de control. Su limitación es la ausencia de un grupo control, la presencia de aproximadamente $10 \%$ de sujetos sin ventana acústica, el ser desarrollado en 
una institución de alta complejidad, con operadores de alta experiencia para una técnica diagnóstica que es operador dependiente y, por lo tanto, no es necesariamente aplicable a otras instituciones con sobre carga asistencial en urgencia.

En conclusión, la ST es factible de realizar en un centro médico complejo, es segura y podría ser implementada como apoyo terapéutico en nuestros centros de salud a nivel nacional.

\section{Referencias}

1. 10 primeras causas de muerte en Chile entre el 20002010. Disponible en: http://www.deis.cl/estadisticasmortalidad/ [Consultado el 15 de noviembre de 2012].

2. Burke JF, Lisabeth LD, Brown DL, Reeves MJ, Morgenstern LB. Determining stroke's rank as a cause of death using multicause mortality data. Stroke 2012; 43: 2207 11 .

3. The National Institute of Neurological Disorders and Stroke rt-PA Stroke Study Group. Tissue plasminogen activator for acute ischemic stroke. N Engl J Med 1995; 333: 1581-7.

4. Hacke W, Kaste M, Bluhmki E, Brozman M, Dávalos A, Guidetti D, et al. Thrombolysis with alteplase 3 to 4-5 hours after acute ischemic stroke. N Engl J Med 2008; 359: 1317-29.

5. Del Zoppo GJ. Trombolitic therapy in treament of stroke. Drugs 1997; 54 (3): 90-9.

6. Broderick JP, Palesch YY, Demchuk AM, Yeatts SD, Khatri P, Hill MD, et al. Endovascular therapy after intravenous t-PA versus t-PA alone for stroke. N Engl J Med 2013; 368: 893-903.

7. Ciccone A, Valvassori L, Nichelatti M, Sgoifo A, Ponzio $\mathrm{M}$, Sterzi R, et al. Endovascular treatment for acute ischemic stroke. N Engl J Med 2013; 368: 904-13.

8. Kidwell CS, Jahan R, Gornbein J, Alger JR, Nenov V, Ajani $Z$, et al. A trial of imaging selection and endovascular treatment for ischemic stroke. N Engl J Med 2013; 368: 914-23.

9. Parsons M, Spratt N, Bivard A, Campbell B, Chung K, Miteff F, et al. A randomized trial of tenecteplase versus alteplase for acute ischemic stroke. N Engl J Med. 2012; 366: 1099-107.

10. von Kummer R, Albers GW, Mori E, Chabriat H, Chang KC, Dávalos A, Ford G, et al. The Desmoteplase in Acute Ischemic Stroke (DIAS) clinical trial program. Int J Stroke 2012; 7: 589-96.

11. Alexandrov AV, Molina CA, Grotta JC, Garami Z, Ford SR, Álvarez-Sabin J, et al. Ultrasound-enhanced systemic thrombolysis for acute ischemic stroke. N Engl J Med 2004; 351: 2170-8.

12. Eggers J, Koch B, Meyer K, König I, Seidel G. Effect of ultrasound on thrombolysis of middle cerebral artery occlusion. Ann Neurol 2003; 53: 797-800.

13. Trubestein G, Engel C, Etzel F, Sobbe A, Cremer H, Stumpff U. Thrombolysis by ultrasound. Clin Sci Mol Med Suppl 1976; 3: 697s-98s.

14. Saqqur M, Shuaib A, Alexandrov AV, Hill MD, Calleja $\mathrm{S}$, Tomsick T, et al. Derivation of transcranial Doppler criteria for rescue intra-arterial thrombolysis: multicenter experience from the Interventional Management of Stroke study. Stroke 2005; 36: 865-8.

15. Molina CA, Ribo M, Rubiera M, Montaner J, Santamarina E, Delgado-Mederos R, et al. Microbubble administration accelerates clot lysis during continuous $2-\mathrm{MHz}$ ultrasound monitoring in stroke patients treated with intravenous tissue plasminogen activator. Stroke 2006; 37: 425-9.

16. Brott T, Adams HP Jr, Olinger CP, Marler JR, Barsan WG, Biller J, et al. Measurements of acute cerebral infarction-a clinical examination scale. Stroke 1989; 20: 864-70.

17. Goldstein LB, Bartels C, Davis JN. Interrater reliability of the NIH Stroke Scale. Arch Neurol 1989; 46: 660-2.

18. Albanese MA, Clarke WR, Adams HP Jr, Woolson RF. Ensuring reliability of outcome measures in multicenter clinical trials of treatments for acute ischemic stroke. The program developed for the Trial of Org 10172 in Acute Stroke Treatment (TOAST). Stroke 1994; 25: 1746-51.

19. Hoppe H, Brunser A, Cárcamo DA, Díaz V, Lavados PM, Manterola JL, et al. NIHSS en español. Disponible en: http://nihss-spanish.trainingcampus.net [Consultado el 10 de marzo de 2013].

20. Brunser AM, Lavados PM, Cárcamo DA, Hoppe A, Olavarría V, Díaz V, et al. Additional information given to a multimodal imaging stroke protocol by transcranial Doppler ultrasound in the emergency room: a prospective observational study. Cerebrovasc Dis 2010; 30: 260-6.

21. Brunser AM, Silva C, Cárcamo D, Muñoz P, Hoppe A, Olavarría VV, et al. Transcranial Doppler in a HispanicMestizo population with neurological diseases: a study of sonographic window and its determinants. Brain Behav 2012; 2: 231-6.

22. Demchuk AM, Burgin WS, Christou I, Felberg RA, Barber PA, Hill MD, et al. Thrombolysis in Brain Ischemia (TIBI) transcranial Doppler flow grades predict clinical severity, early recovery, and mortality in patients treated with intravenous tissue plasminogen activator. Stroke 2001; 32: 89-93. 
23. Rankin J (May 1957). "Cerebral vascular accidents in patients over the age of 60. II. Prognosis". Scott Med J 2 (5): 200-15.

24. Farrell B, Godwin J, Richards S, Warlow C, "The United Kingdom transient ischaemic attack (UK-TIA) aspirin trial: final results". J Neurol Neurosurg Psychiatry 1991; 54 (12): 1044-54.

25. van Swieten JC, Koudstaal PJ, Visser MC, Schouten HJ, van Gijn J. Interobserver agreement for the assessment of handicap in stroke patients. Stroke 1988; 19 (5): 604-7.

26. RG Newcombe, Two-sided confidence intervals for the single proportion: Comparison of seven methods. Sta- tistics in Medicine 1998; 17: 857-72.

27. Rha JH, Saver JL. The impact of recanalization on ischemic stroke outcome: a meta-analysis. Stroke 2007; 38: 967-73.

28. Ricci S, Dinia L, Del Sette M, Anzola P, Mazzoli T, Cenciarelli S, et al. Sonothrombolysis for acute ischaemic stroke. Cochrane Database of Systematic Reviews. Issue 7, 2013.

29. Rivas R, Klapp C, Abarca MC, Bustos L, Manosalva M, Merino C, et al. Sonothrombolysis in Araucanía, Chile: thrombolytic treatment in acute ischemic stroke in a new stroke center. Cerebrovasc Dis 2013; 35 (suppl. 2): 65-6. 